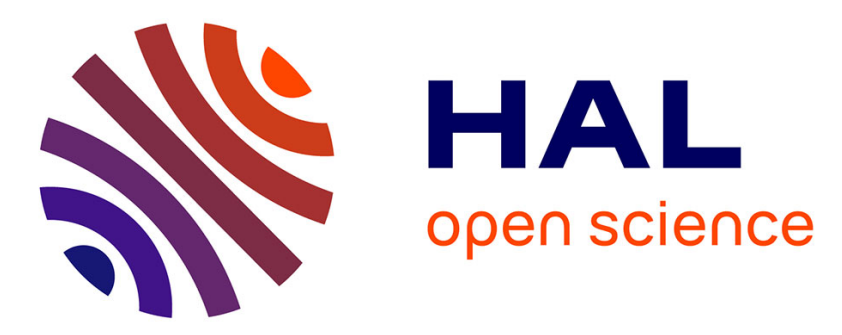

\title{
Une typologie de maires entrepreneurs politiques, comme aide au conseil dans les mairies
}

David Huron

\section{To cite this version:}

David Huron. Une typologie de maires entrepreneurs politiques, comme aide au conseil dans les mairies. Politiques et Management public, 2001, 19 (2), p. 63-81. hal-00493580

\author{
HAL Id: hal-00493580 \\ https://hal.science/hal-00493580
}

Submitted on 11 Feb 2016

HAL is a multi-disciplinary open access archive for the deposit and dissemination of scientific research documents, whether they are published or not. The documents may come from teaching and research institutions in France or abroad, or from public or private research centers.
L'archive ouverte pluridisciplinaire HAL, est destinée au dépôt et à la diffusion de documents scientifiques de niveau recherche, publiés ou non, émanant des établissements d'enseignement et de recherche français ou étrangers, des laboratoires publics ou privés. 


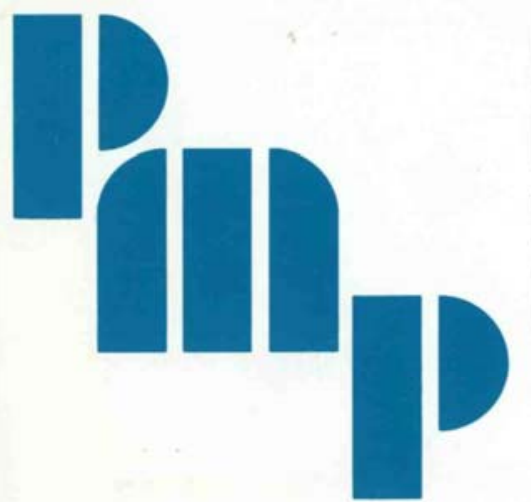

\section{POLITIQUES} ET

MANAGEMENT PUBLIC

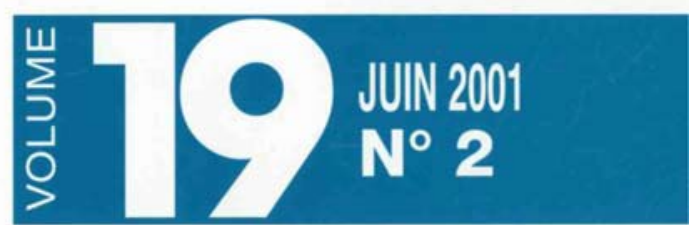

Gérard MARTIN et Amédine RUFFIOT

"La commande d'évaluation de politiques sociales territoriales, entre mythes et apprentissages "

\section{Sandrine KOPEL}

"Le déroulement de carrière des " surdiplômés " de la fonction publique : propositions pour une gestion différenciée *

Alexandre SINÉ

« Responsabilité politique : de la fin du modèle weberien

aux nouvelles figures de légitimation de l'action publique "

David HURON

« Une typologie de maires entrepreneurs politiques comme aide au conseil dans les mairies *

Laurent CARNIS

" L'apport de l'analyse économique au contrôle des excès de vitesse $n$

Carine CATELIN

"La responsabilité dans la gestion des investissements - rôle du contrôle et de l'apprentissage organisationnel - : le cas de France Télécom "

Jean de KERVASDOUÉ

"Lieux communs et idées fausses en politique de santé "

HURON D., 2001, « Une typologie de maires entrepreneurs politiques comme au aide au conseil dans les mairies ", Politiques et Management Public, vol.19, n², p.63-81. 


\section{UNE TYPOLOGIE DE MAIRES ENTREPRENEURS POLITIQUES COMME AIDE AU CONSEIL DANS LES MAIRIES}

\section{David HURON*}

Résumé

De nombreuses contributions comparent la gestion de la commune à celle de l'entreprise. Cette démarche est reprise pour analyser la fonction de maire par analogie à celle de chef d'entreprise. Pourtant cette approche souffre de ne pas prendre en compte simultanément les dimensions politiques et managériales.

En se fondant sur les qualités effectivement possédées et les risques encourus par les maires sur trois champs d'action, cet article a pour objectif de mettre en évidence une typologie de maires entrepreneurs politiques.

Ce travail s'appuie empiriquement sur une enquête par questionnaire effectuée en janvier 1997 auprès des maires de communes françaises de plus de 20000 habitants (hors Paris et départements d'outre-mer).

* CRIFP-IDEFI (Centre de Recherche en Ingénierie Financière et Finances Publiques - Institut du Droit et de l'Economie de la Firme) à Sophia-Antipolis.

Revue POLITIQUES ET MANAGEMENT PUBLIC, Volume 19, n², juin 2001.

(C) Institut de Management Public - 2001. 
Introduction
La fin des années 1960 et le début des années 1970 ont vu se développer en France un ensemble de travaux portant sur le système politico-administratif local sous l'impulsion du Centre de Sociologie des Organisations [Crozier M., 1967 ; Gremion P., 1970]. Certains auteurs, proches de ce courant [BecquartLeclercq J., 1976; Thoenig J.-C., 1975], se sont plus particulièrement intéressés à l'acteur " maire ". Ces études sont relayées par des travaux plus récents portant sur le gouvernement local [Borraz O., 1994]. Par ailleurs, la fonction de maire a fait l'objet depuis le milieu des années 1980 de développements théoriques consistant à la rapprocher de la fonction de chef d'entreprise ${ }^{1}$ [Guengant A., 1985; Klopfer M., 1986; Le Duff R. et Rigal J.-C., 1996]. II semble néanmoins que les contributions consacrées aux comparaisons entre la commune et l'entreprise n'aient pas apporté de réponse satisfaisante à l'analyse de la fonction. La spécificité d'une commune et plus généralement d'une collectivité territoriale nécessite en effet d'adopter une démarche qui lui est propre et non de sacrifier aux modes de gestion [Gibert P., 1990]. De manière générale, deux logiques sous-tendent l'action du maire. En premier lieu, une logique entrepreneuriale guide le responsable politique dans ses décisions. II pourra perturber l'équilibre existant en adoptant des stratégies de rupture, ou stabiliser l'organisation après une période de changement en endossant une démarche d'accumulation (c'est-àdire en s'adaptant à la contrainte). En second lieu, le maire obéit à une logique politique 2 qui accroît sa dépendance à des groupes de pression. Ces deux logiques, usuellement séparées constituent les fondements du concept de maire entrepreneur politique. Pour autant, la démarche ne saurait se réduire à l'introduction d'une dimension politique dans une analyse du maire à l'image du chef d'entreprise. Les propos qui suivront s'appuieront dès lors sur ces deux logiques intégrées et indissociables.

L'entrepreneur politique est défini par Guillaume Payen comme " un agent de déséquilibre qui remet en cause l'ordre et les habitudes en vigueur. C'est un type particulier d'acteur jouant un rôle innovant et structurant" [1987, p.72]. L'élaboration d'un projet original ou la remise en cause d'une partie des représentations et des normes régissant l'activité managériale, ne permet pas de distinguer le rôle de l'entrepreneur de celui du politique. La mise en œuvre d'un projet ou l'institution d'un nouveau cadre ainsi que la découverte des ressources et contraintes composant l'environnement témoignent également de cette mixité. Cette acception de l'entrepreneur politique est particulièrement adaptée à la fonction mayorale. Trois champs d'analyse semblent mettre en lumière le concept de maire entrepreneur politique. Ainsi, l'action du maire peut être caractérisée par des positionnements sur les axes équilibre/déséquilibre de l'organisation, par une prise en compte ou non de l'agenda politique, ainsi que par l'existence/absence de partenariat (cf. figure 1).

1 En 1986, la Revue Française de Finances Publiques dans son $n^{\circ} 13$ consacrait un dossier entier à la comparaison entre gestion des communes et des entreprises.

2 Selon les deux sens: action dans le cadre d'un groupe d'hommes organisés et action orientée vers l'organisation publique. 
Figure 1

Le positionnement des maires sur les champs d'action

Agent de
déséquilibre

Dès lors, le maire entrepreneur politique (MEP), dont l'action est limitée dans le temps, est un acteur coopératif, jouant un rôle innovant et structurant dans l'environnement local. II est à la fois agent de déséquilibre sur le champ de l'équilibre organisationnel, partenaire sur le champ du partenariat et manager de l'agenda politique sur le champ de l'équilibre politique ${ }^{1}$. L'absence d'au moins un de ces trois attributs révèle un défaut de représentation sur les champs d'action concernés. Dans ces conditions, des risques ${ }^{2}$ peuvent émerger et mettre en difficulté le maire et sa commune.

Les

déterminants de la typologie : les risques issus d'un défaut de représentation sur les champs d'action du maire
Trois types de situation peuvent caractériser les risques du maire entrepreneur politique. L'étude des champs d'action peut d'abord révéler l'absence d'une des qualités nécessaires au dirigeant de la commune. Le risque sera alors relatif à ce champ d'action qui sera proportionnellement déficitaire par rapport aux deux autres. Par ailleurs, deux qualités peuvent également faire défaut. Dans ce cas, non seulement les risques apparaîtront au niveau des champs d'action que le maire ne maîtrise pas, mais aussi au niveau de celui sur lequel le maire axe toute son énergie. Ainsi, à terme, l'effet recherché risque d'être dénaturé, le maire s'étant trop engagé sur ce champ.

Enfin, lorsque le maire ne possède aucune des qualités nécessaires, la multiplicité des risques rend caduque toute tentative de prévention. Le maire n'est pas dans ce cas un entrepreneur politique dans la mesure où il ne possède pas au moins une des qualités correspondant à un champ d'action.

\footnotetext{
1 Par extension le maire sera qualifié de MEP agent de déséquilibre, manager de l' agenda politique ou partenaire si celui-ci ne possède qu'une seule qualité.

2 Le risque est ici envisagé selon une connotation plutôt négative et non selon une vision duale du concept [Padioleau J.-G., 1999].
} 
Les développements suivants ont pour objet de dresser un état des lieux.des types de risque encourus en fonction du positionnement des maires sur chacun des champs d'action. Pour visualiser les diverses situations de maire entrepreneur politique, la figure 2 définit en fonction des qualités possédées ou non les types de problèmes qui risquent d'apparaître. Pour autant, cette "représentation" traduit moins un modèle rigide qu'un "guide " permettant de mettre en évidence des relations entre le positionnement des maires sur chacun des champs d'analyse et les risques ${ }^{1}$ les plus fréquemment rencontrés [Bouquin H., 1996, p. 98]. De manière générale, deux types de risque apparaissent sur chacun des champs d'action.

Figure 2

Les risques encourus par les maires

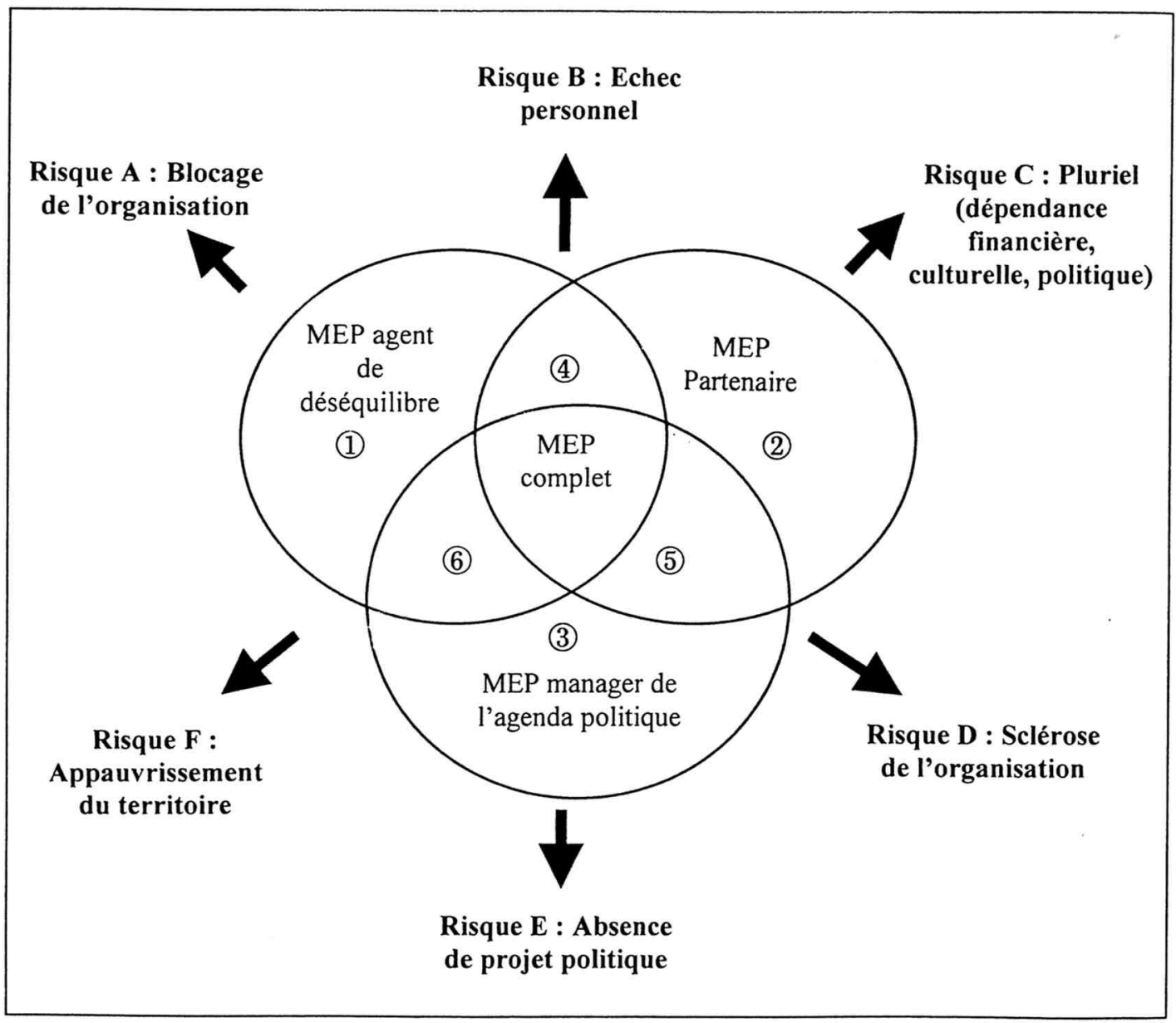

1 Sur le graphique, ces risques prennent les lettres A à F. Cette convention de notation sera reprise dans les commentaires qui suivront. 


\section{Les risques sur le champ de l'équilibre organisationnel}

Le continuum de l'équilibre organisationnel marque à chaque extrémité un type de risque particulier : soit le maire est uniquement agent de déséquilibre et ignore les deux autres champs d'action (risque A), soit au contraire le maire ignore le champ de l'équilibre organisationnel tout en possédant les qualités de partenaire et de manager de l'agenda politique (risque D).

- Le risque A: un excès de déséquilibre et un risque de blocage de l'organisation

Le changement impulsé par les élus induit bien souvent une capacité d'adaptation de la part des acteurs concernés. Fondée sur "une évaluation multidimensionnelle des capacités actuelles et futures" de la commune, l'adaptation fait plus référence à la notion de qualité de changement qu'à celle d'une quantité $^{1}$ [Véran L., 1991, p. 59]. Or, la situation où le maire remet en question des fonctionnements routiniers pousse bien souvent les fonctionnaires à résister aux changements. Un accompagnement de ces derniers est donc nécessaire, afin d'éviter les blocages organisationnels. Toutefois un excès de déséquilibre met en danger la structure municipale dans la mesure où peuvent apparaître des dysfonctionnements plus graves: désorganisations, grèves des fonctionnaires, services non réalisés ou mal effectués, absentéisme important...

Ces problèmes sont d'autant plus préoccupants que les champs du partenariat et de l'équilibre politique ne sont pas occupés par le maire. Or, la résolution de situations où il existe une hostilitè du personnel nécessite d'avoir recours aux deux autres champs d'action. Les risques $B$ et $F$ définis plus loin constituent donc des menaces venant s'ajouter à celle que nous venons de décrire.

- Le risque $D$ : une absence de déséquilibre et un risque de sclérose de l'organisation

L'absence de changement organisationnel implique une certaine inertie des organisations a fortiori lorsqu'elles sont publiques. L'administration est souvent qualifiée de bureaucratique. Cette bureaucratie s'installe ou se pérennise en cas d'absence d'action du maire sur le champ de l'équilibre organisationnel. L'absence de déséquilibre entretient alors un "caractère de prévisibilité " qui, poussé à l'extrême, peut avoir des effets très néfastes sur l'action impulsée par le maire.

Progressivement s'installe une "différenciation marquée des rôles entre les acteurs sociaux". Cette situation "crée une division verticale dominante $d u$ travail entre les dirigeants et les gouvemés " [Padioleau J.-G., 1986, p.171]. Cette institutionnalisation des rôles aboutit donc à une sclérose de l'organisation liée à son degré d'inertie.

1 Lucien Vèran souligne que la qualité du changement est essentielle pour l'adaptation. En revanche, la quantité fait plus référence à la régulation. 
Les raisons de cette paralysie de la structure municipale peuvent être recherchées au niveau de l'absence d'objectifs liés à une meilleure performance des services. La routine et l'habitude favorisent ainsi progressivement l'absence de qualité dans l'exécution des missions. Les dysfonctionnements issus de la non-qualité sont accentués par l'existence d'une bureaucratie qui, même si elle constitue un problème organisationnel "programmable" [Duran P. et Thoenig J.-C., 1996, p. 598], constitue un obstacle supplémentaire sur le champ de l'équilibre organisationnel.

\section{Les risques sur le champ du partenariat}

Le champ du partenariat constitue également un continuum borné à chaque extrêmité par un type de risque. Le maire peut d'abord n'être présent que sur le champ d'action partenarial (risque $C$ ). II peut à l'inverse ne pas investir l'axe du partenariat et risquer l'isolement (risque F).

\section{- Le risque $\mathrm{C}$ : un excès de partenariat et des risques pluriels}

Cette situation met en exergue l'absence de représentation du maire sur les champs des équilibres politiques et organisationnels (risques $D$ et $B$ ). Le maire consacre alors l'essentiel de son action au niveau de la mise en œuvre du partenariat. Pour autant, lorsque ce dernier est utilisé à trop forte dose, il est inévitable qu'un certain nombre de dysfonctionnements apparaissent. La multiplicité des partenariats implique des relations avec une pluralité d'acteurs (entreprises, opérateurs de services urbains, divers groupes d'intérêt, autres collectivités locales, Etat...). Dès lors, les orientations des maires doivent prendre en considération un "émiettement des centres de décision" [Wachter S., 1995, p. 33].

De par un contrôle devenu difficilement réalisable, ce système rend les décisions municipales incertaines. Cette incertitude est liée au nombre important des partenaires du maire, qui sont autant de risques de ruptures de coopération au sens où chacun essaie de ne pas respecter l'accord initial. Dans la mesure où les partenaires revendiquent leur indépendance par rapport au maire, on observe une dilution du risque qui "devient pluriel" [Marfaing O. et Spindler J., 1998, p. 326]. Cette pluralité peut être illustrée concrètement par une perte de contrôle financier, culturel ou politique suivant le type de partenaires du maire ${ }^{1}$.

- Le risque $F$ : une absence de partenariat et un risque d'appauvrissement $d u$ territoire

Si rien n'oblige un maire à utiliser le partenariat, certains arguments l'encouragent fortement en ce sens. Cet argumentaire, essentiellement fondé sur les risques encourus, concerne plus particulièrement le thème générique

1 La constitution de communautés urbaines ou d'agglomération est souvent perçue et vécue comme une perte de pouvoir et de contrôle par les maires des communes périphériques à la ville centre. 
de l'appauvrissement du territoire. Dans cette optique, le risque peut être décliné selon quatre pistes [Orange G., 1992, p. 240]. D'abord, l'absence de partenariat favorise un risque de précarisation dans la mesure où il existe une dualité entre centre riche et appauvrissement accéléré de la périphérie. Ensuite, il existe un risque de dépeuplement liè à l'absence de certaines infrastructures ou services que la commune seule ne peut financer. Cette perte de population constitue le départ d'un cercle vicieux car à l'origine d'une diminution de la DGF. Un troisième risque lié à l'absence de partenariat touche la congestion de la commune. Ainsi, les communes-centres d'agglomérations sont seules à financer des effets externes négatifs (dégradation du cadre de vie, système de communication saturé,...), que les communes environnantes pourraient financer en cas de partenariat. Enfin, l'absence de communication liée au défaut de coopération fait peser sur la commune un risque d'isolement. De manière générale, les risques que nous venons de développer font apparaître une perte, quelle qu'en soit sa nature, pour la commune.

\section{Les risques sur le champ de l'équilibre politique}

Le troisième champ d'action, relatif à l'équilibre politique, comporte lui aussi des risques liés à la position du maire vis-à-vis de celui-ci. La problématique de ce champ d'action peut se résumer en une question: "le politique influence-t-il la décision de par ses idées politiques, ou est-il influencé par ce que pensent (et votent) les électeurs ?" [Dahl R., 1971, p. 113]. Le risque électoral peut toucher l'élu lorsque ce dernier ne prend pas en compte l'agenda politique de sa commune (risque B). Au contraire lorsque l'élu a l'œil rivé sur son agenda, il risque de dénaturer son action politique et de s'éloigner du projet initialement élaboré (risque E).

- Le risque $E$ : l'éloignement du projet politique initial par une trop forte prise en compte de l'agenda politique

Cette situation implique que les maires n'aient pas de manière générale recours au partenariat et ne visent pas à changer l'organisation. De fait, les risques $D$ et $F$ concernent également cette catégorie. De surcroît, une surreprésentation de la prise en compte de l'agenda politique met en évidence d'autres risques. Comme le souligne Serge Wachter, on assiste au "triomphe de l'issue politics", c'est-à-dire que les décisions sont assujetties "aux demandes des corporations qui s'échangent contre des assurances de soutien convertibles en promesses de vote "[1998, p. 174]. Cette stratégie n'est pas une nouveauté dans la mesure où pour gagner des élections, l'art de la politique consiste à effectuer des promesses aux coalitions électoralement les plus représentatives [Salancik G.R. et Pfeffer J., 1977, p. 483], y compris au sein de la structure municipale [Dion S., 1986]

Cette multiplication des concessions effectuées peut légitimement poser le problème de la remise en cause du projet politique initial. En effet, la "confusion des centres de décisions" et "la collusion des intérêts" sont monnaie courante [Vieilleville J., 1994, p.102]. Bien souvent l'acceptation de "compromis complexes" contre une assurance de soutien suffit à l'exercice 
du mandat [Charreaux G., 1997, p.47]. Ainsi, il peut être électoralement plus intéressant pour un maire de satisfaire ses concitoyens (baisse des impôts, création de nouveaux services), quitte à créer un déficit parfois important au niveau du budget communal.

- Le risque $B$ : Une absence de prise en compte de l'agenda politique et un risque électoral

L'absence de prise en compte de l'agenda politique est révélatrice de la volonté à appliquer un projet politique initialement défini. Pour autant, le fait de ne pas accepter les compromis, notamment dans le cas où la population exprimerait certaines attentes, peut induire un risque électoral. Si les décisions autoritaires garantissent certains bénéfices à leurs auteurs, elles les éloignent parallèlement de l'exercice de mandats futurs [Moe T.M., 1995]. En effet, si un maire a été à l'origine élu sur un programme, l'application rigoureuse de ce dernier ne garantit pas la satisfaction de ses concitoyens.

En outre, le projet politique peut être décalé des préoccupations des populations. Un choix paraissant approprié à un moment donné peut devenir obsolète, voire improductif avec le temps. La perception négative par la population de ce type de décision peut influencer de manière décisive les chances de réélection du maire. Enfin, l'absence de réalisation concrète ou de perception de projet politique clair est également susceptible d'influer négativement sur le scrutin municipal. Si la date de livraison d'un équipement ne correspond pas à la fin d'un mandat, sa réalisation ne sera pas imputée au maire et ne lui procurera donc pas de profit politique. Cette constatation s'appuie sur les théories du cycle électoral et du cycle d'investissement [Le Duff R. et Orange G., 1996].

Méthode d'investigation 1
Afin de mesurer la nature des risques encourus par les maires, une enquête quantitative a été menée sur la base d'un questionnaire volontairement réduit à une trentaine de questions. Celui-ci a été adressé à tous les maires de commune de plus de 20000 habitants hors Paris et départements d'outremer, soit 400 communes selon le guide pratique de la décentralisation ${ }^{2}$. La structure du questionnaire reprend les thèmes de l'étude. Les informations obtenues ont fait l'objet de certains traitements statistiques.

\section{Nature et traitement des informations obtenues}

Le questionnaire est articulé autours de quatre thèmes. En premier lieu, un descriptif général du profil des maires permet de préciser les disparités observées entre élus et de souligner les caractéristiques des villes étudiées : taille, région, configuration de la commune (commune-centre, périphérique, de

1 Des éléments plus approfondis sur l'enquête et ses résultats peuvent être trouvés dans des travaux antécédents [Huron D., 1999].

2 Le choix des villes moyennes et de grande importance est la résultante des thèmes liés à la problématique [Busson-Villa F., 1999]. Les questionnaires ayant été envoyés en octobre 1996 (soit plus d'un an après le scrutin municipal), l'ensemble des retours se sont étalés jusqu'en février 1997. 
région parisienne ou de province). II contribue également à étudier des facteurs liés aux parcours personnels des maires avant leur élection par des variables telles que la profession, le nombre de mandat de maire effectué, ou l'expérience municipale avant l'élection. En outre, ce descriptif permet de mettre en évidence la carrière politique du maire par le cumul de mandat, le type de mandat cumulé, la présidence de structure intercommunale et l'appartenance politique.

Le second thème tente de mesurer le champ de l'équilibre organisationnel par la capacité du maire à devenir un agent de déséquilibre. Cette capacité est en premier lieu liée à l'impulsion d'un déséquilibre structurel. Ce dernier peut prendre la forme d'une modification structurelle de l'organigramme (par division, fonction ou autre) ou d'une mise en place de projet de commune. II peut également apparaître à travers l'introduction d'innovations tant dans l'organisation municipale et la gestion ${ }^{1}$ que dans les relations avec les partenaires (autres collectivités territoriales, entreprises) ou les fonctionnaires territoriaux, ou encore dans les services publics offerts à la population. Le déséquilibre peut en second lieu prendre forme dans les relations entre élus et administratifs. Celui-ci est rendu visible par le type de relations entretenu entre le maire et le secrétaire général, par leur fréquence de rencontres, et par le type de suivi qu'accorde le maire à un projet.

L'étude du champ de l'équilibre politique nécessite de connaître la capacité du maire à agir comme un manager de l'agenda politique. Dans cette optique, le maire posséderait un comportement électoraliste. Celui-ci peut être mis en évidence par des variables comme la proximité des échéances des actions que le maire a entreprises, ses priorités fiscales, ainsi que les domaines qu'il juge prioritaires. Parallèlement, le maire chercherait à laisser une empreinte de son passage à la tête de sa municipalité. C'est pourquoi à travers les investissements et les cibles de communication qu'il envisage, il recherche le développement économique de son territoire. Le dernier thème abordé dans le questionnaire est relatif au partenariat. Le maire jongle en effet entre un partenariat de gestion intégré impliquant un véritable suivi et un partenariat d'investissement plutôt axé sur une participation ponctuelle.

Le degré de coopération se retrouve tout d'abord dans la relation de l'élu avec ses administrés. Ainsi, les enquêtes d'opinion ou les réunions publiques impliquent une participation des habitants beaucoup moins active que les comités de quartier par exemple. De même, le degré de coopération est visible dans les relations avec les partenaires privés (utilisation de SEM ou de gestion déléguée) et dans les relations avec d'autres partenaires publics suivant la nature de l'intercommunalité.

Le traitement des questionnaires a consisté en premier lieu à effectuer des tris à plat et des tris croisés. La nature des données qui ont été recueillies implique d'avoir eu recours à des tests non paramétriques ${ }^{2}$. En fonction des champs d'action, des cartes factorielles ont été construites selon le principe de l'analyse factorielle en composantes multiples afin de percevoir visuellement les relations de dépendance entre variables. Sur chacune des

1 Notamment par l'introduction de nouveaux outils.

2 Ainsi, le test du khi-deux de Pearson nous a permis d'analyser la structure de la dépendance entre les variables et d'en faire ressortir les traits principaux [Bouroche J.-M. et Saporta G., 1993]. 
cartes factorielles, une dichotomie a été effectuée en deux classes représentant la possession ou non de la qualité (partenaire, agent de déséquilibre ou manager de l'agenda politique). Le positionnement des maires sur les champs d'action constitutifs du MEP (à partir des trois cartes factorielles) a permis de construire une typologie de maires.

\section{Représentativité de l'échantillon}

Le taux de réponses exploitables s'est révélé particulièrement élevé $(46,5 \%$ de la population). Le cautionnement du secrétaire général de l'Association des Maires de France enjoignant à ses collègues de répondre explique en grande partie que 186 d'entre eux aient répondu. Bien que présenté comme s'adressant directement aux maires, il semblait probable qu'une grande partie d'entre eux, ayant diverses fonctions électives et/ou gouvernementales, délèguent le questionnaire par manque de temps à leur entourage professionnel proche. C'est pourquoi, à la fin du questionnaire, il était demandé l'identité du ou des répondants afin d'introduire dans l'analyse d'éventuels biais dus à des différences entre les catégories de répondants. En réalité, une majorité de maires (y compris parmi les plus grandes villes) ont directement répondu, ce qui constitue les trois-quarts des réponses exploitables $(75,3 \%)$. Le quart restant se partage entre les directeurs de cabinet $(20,4 \%)$ et les secrétaires généraux $(4,3 \%)$. II est à noter que certains questionnaires ont été remplis par au moins deux de ces acteurs.

Bien que le risque de non-représentativité de l'échantillon soit limité du fait du taux de réponse important, une comparaison entre la répartition nationale et celle de l'échantillon a été effectuée lorsque les chiffres étaient disponibles. Ainsi, les résultats sont comparables tant du point de vue de la taille des communes que de celui de la répartition géographique ou de l'appartenance politique des maires.

\section{Vers une typologie de maires entrepreneurs politiques?}

II convient à présent de se pencher sur les différences de profil issues des trois champs d'action définissant le MEP. La construction d'une typologie dépend étroitement de la situation des maires au moment de l'enquête et du type de variables pris en compte. Ainsi, les cartes de champs d'action des maires ont permis de mettre en évidence différents types de maires (Cf. annexe 1).

Chaque maire fait l'objet d'un positionnement dichotomique sur ses trois champs d'action. La répartition quantitative de la population au sein des différentes catégories s'est effectuée de manière très inégale comme le montre la figure 3 , certaines catégories de maires représentant moins de $10 \%$.

Dans une optique d'aide au conseil des élus locaux, la typologie proposée se fonde sur l'absence de qualité observée. Ainsi, quatre catégories ont été mises en évidence : les maires non partenaires, les maires non agents de déséquilibre, les maires non manager des échéances et les MEP complets. Les développements suivants reprennent en détail chacun d'entre eux. 


\section{Figure 3}

Répartition quantitative des différents types de maires

(8) Maire non entrepreneur politique $4,3 \%$

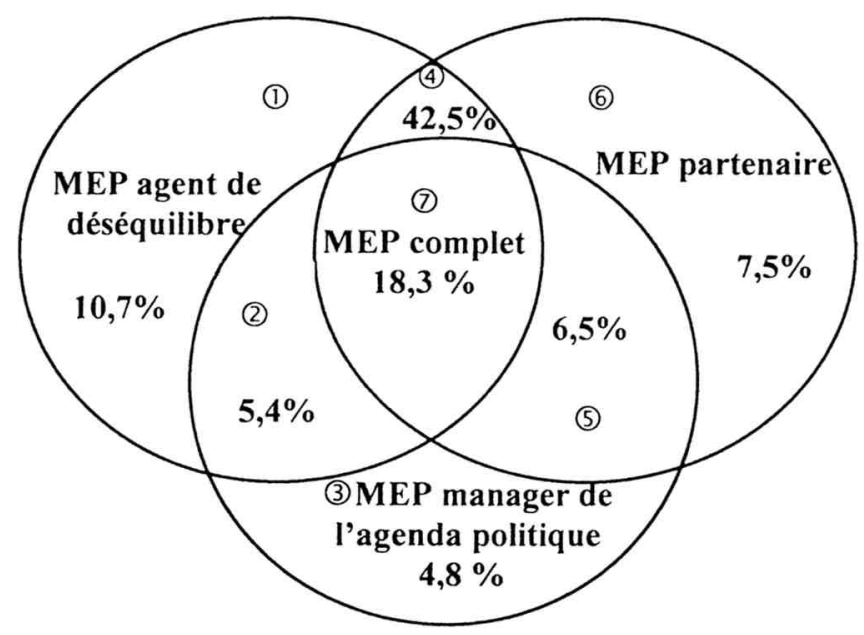

\section{- Les maires non agents de déséquilibre}

Cette catégorie $\left(23,6 \%\right.$ des maires) ${ }^{1}$ est caractérisée par une propension à innover beaucoup moins forte que celle observée pour l'ensemble de la population étudiée. Ce constat se retrouve dans tous les domaines (services publics, organisation et gestion, partenariats). Les maires non agents de déséquilibre semblent plus modifier leur structure organisationnelle par l'introduction de nouveaux éléments par rapport à leurs collègues. Par contre le recours aux projets de communes est beaucoup moins utilisé. La nature de la relation que les maires entretiennent avec leurs principaux collaborateurs relève plus du contrôle que de la délégation. En effet près de huit d'entre eux sur dix ont un entretien quotidien avec leur secrétaire général, contre environ $47 \%$ pour l'ensemble des maires interrogés.

Les villes de taille plus modeste (de 20000 à 49999 habitants) sont mieux représentées dans cette catégorie. Les maires, dans cet échantillon, y effectuent majoritairement leur premier mandat (45,5\% d'entre eux). Ces nouveaux élus ont plus particulièrement ressenti un besoin de formation en ressources humaines et en comptabilité. Au niveau du cumul de mandats, ces élus semblent privilégier celui de conseiller général (contre celui de parlementaire pour l'ensemble des maires). Les maires non agents de déséquilibre privilégient le court terme par rapport au moyen terme, ce qui se

1 Ce chiffre s'obtient en additionnant les chiffres correspondant aux types de maires (3) (5) (6) (8) sur la figure 3. 
traduit par une préférence pour les investissements de proximité au détriment des investissements tournés vers les entreprises. II faut noter en outre que les liens traditionnels entre le maire et ses administrés (réunions, comités de quartiers, mairies annexes) sont systématiquement moins représentés dans cette catégorie. Cette frilosité quant à une gestion partenariale de la commune se retrouve à plusieurs niveaux; on observe une sur-représentation des syndicats de commune, un déficit d'utilisation des SEML par rapport à l'ensemble des communes répondantes ainsi qu'une certaine prudence dans l'utilisation de la gestion déléguée dans des domaines comme les ordures ménagères ou les transports urbains. Cette prudence dans les formes de coopération intercommunale utilisées peut s'expliquer par le défaut de maîtrise de ces dernières. Dans cette catégorie, $53,6 \%$ ne président pas de structure intercommunale contre $42,3 \%$ pour l'ensemble des maires répondants.

\section{- Les maires non manager de l'agenda politique}

La maîtrise de l'agenda politique semble paradoxalement faire défaut à la majorité des maires de l'échantillon (65\% d'entre eux $)^{1}$. La complexité de la situation municipale implique une forte incertitude, ce qui rend difficile les tentatives de contrôle de l'agenda politique [Thoenig J.-C., 1995]. La pérennité de l'action du maire constitue l'enjeu principal de la possession de cette qualité. Cette catégorie semble souffrir d'une non reconnaissance de la population au moment des échéances électorales, alors qu'un projet dépassant le cadre du mandat municipal a été mis en œuvre. Cette situation peut être illustrée par le fait que les maires non manager de l'agenda politique sont plus nombreux à agir à moyen terme. En outre, il communiquent et privilégient les investissements vers les entreprises dans le but de développer l'économie locale.

Les maires non manager de l'agenda politique paraissent détenir plus d'expérience; les nouveaux élus sont proportionnellement moins nombreux $(32,2 \%$ contre $37,6 \%)$. Cette expérience se traduit par un cumul systématique pour près de la moitié d'entre eux d'un mandat de parlementaire. Les maires de cette catégorie orientent leur gestion au quotidien vers une confiance accordée à leurs collaborateurs au premier rang desquels se trouve le secrétaire général. Bien souvent en effet ce dernier a l'expérience du travail avec l'élu. L'emploi quasi-systématique du projet de commune dans la gestion municipale (pour $81 \%$ d'entre eux) s'accompagne assez souvent d'une liberté de gestion laissée au secrétaire général. Dans cette optique, la confiance accordée par le maire au secrétaire général explique le score moindre de leurs rencontres quotidiennes. Enfin, la gestion partenariale de la commune ne constitue pas une limite pour les maires non manager de l'agenda politique puisqu'ils privilégient la gestion des services par les SEML ainsi que par les structures intercommunales (présidées par le maire dans trois cas sur cinq).

${ }^{1}$ Ce chiffre s'obtient en additionnant les pourcentages des maires de type (1)(4) (6) (8) sur la figure 3 . 


\section{- Le maire non partenaire}

Le maire non partenaire $\left(25,3 \%\right.$ des élus $\left.{ }^{1}\right)$ est caractérisé par un défaut de recours aux liens traditionnels avec la population. Si ce comportement est observable au niveau de l'emploi des comités de quartiers et des mairies annexes, c'est surtout le déficit d'organisation de réunions publiques $(27,3 \%$ des maires contre $73,6 \%$ ) qui illustre plus particulièrement cette situation.

De même, cette prudence dans la gestion partenariale se retrouve dans la nature de la coopération intercommunale ; les districts sont moins représentés alors que sept communes sur dix (contre six pour l'ensemble des répondants) appartiennent à un syndicat. La nature de la gestion des activités de la commune fait également l'objet d'une certaine réticence des maires non partenaires. Ainsi, les gestions déléguées ou effectuées par l'intermédiaire d'une SEML semblent moins utilisées par les élus de cette catégorie par rapport à l'ensemble des répondants.

Les maires non partenaires ont un profil professionnel particulier dans la mesure où les retraités et les chefs d'entreprises sont proportionnellement plus représentés dans cette catégorie au détriment des cadres. Ces éléments sont à mettre en rapport avec la sur-représentation des élus les plus anciens (plus de trois mandats). Ce profil traditionnel se retrouve dans le type de mandat cumulé ; le maire non partenaire est parlementaire dans presque trois cas sur cinq. Les relations entre le maire non partenaire et le secrétaire général sont plus orientées vers le contrôle que vers la coopération sincère et véritable. Si les secrétaires généraux sont plus nombreux en proportion à avoir la responsabilité d'un projet de commune, leur nombre à se réunir quotidiennement avec le maire est sensiblement plus élevé que celui de l'ensemble des communes interrogées. Cette volonté de contrôle se retrouve dans la nature de la collaboration entre les deux acteurs. En effet, le maire non partenaire laisse en majorité peu de marge de manœuvre au secrétaire général ; ce dernier met en œuvre et contrôle alors que le maire définit dès le départ les moyens et les objectifs. Enfin, il semble que cette catégorie soit la plus proche de la gestion traditionnelle "en bon père de famille". De manière générale, la fiscalité locale augmente ou stagne et les entreprises ne constituent pas la cible principale de l'action de ces communes (non seulement au niveau de la fiscalité, mais aussi en matière de communication ou d'investissement tournés vers les entreprises).

\section{- Le maire entrepreneur politique complet}

Ce type de maire entrepreneur politique est ici qualifié de "complet" dans la mesure où chacun des champs nécessaires à la fonction ne semble souffrir d'aucune faiblesse. Cette classe est constituée de trente quatre observations. Si la taille de la commune n'est pas une variable explicative du MEP complet, la configuration de la commune semble en revanche avoir une incidence sur cette catégorie ; les communes périphériques d'lle-de-France sont ainsi moins représentées dans ce groupe par rapport à la répartition globale. La surreprésentation de la région Aquitaine au niveau des MEP complet est la résultante de cette observation. De manière générale, il semble qu'une

1 Chiffre obtenu par addition du nombre des maires de type (1)(2) (3) (8) sur la figure 3 . 
majorité relative des maires entrepreneurs politiques complets se situent au chef-lieu du département. Ces maires sont majoritairement cadres ou ont une profession intellectuelle supérieure. Un seul chef d'entreprise est MEP complet, ce qui semble confirmer la spécificité de la gestion d'une commune par rapport à celle d'une entreprise. Ce constat semble suggérer la pertinence de certains travaux où le maire chef d'entreprise s'est retrouvé dans des difficultés de gestion de par l'amalgame effectué entre les deux types d'organisation [Maury Y., 1997]. Par ailleurs, près des trois quarts de l'effectif de cette catégorie ont été élus depuis 1989. En d'autres termes, les élus les plus anciens paraissent susceptibles de rencontrer ou de cumuler les risques. L'expérience acquise par les dirigeants de communes est également une variable explicative du MEP complet. La moitié des observations indique que le maire a été adjoint avant de prendre lui-même les rênes de la municipalité.

Les trois dimensions du MEP complet sont perceptibles dans les réponses effectuées par les maires de cette catégorie. La dimension agent de déséquilibre est accentuée par de très forts pourcentages tant en raison de l'existence de projets de commune, que du développement de l'innovation organisationnelle ou de la volonté de laisser plus de liberté au secrétaire général. Ce dernier peut d'autant plus s'occuper de la gestion de la commune qu'il est apolitique [Thoenig J.-C. et Burlen K., 1998]. Le partenariat est illustré par l'importance de l'existence de réunions publiques, de comités de quartiers ou de l'utilisation de SEML. Enfin, la dimension manager de l'agenda politique chez le MEP complet est soulignée par un effort fiscal particulier, ainsi que par la construction d'infrastructures nécessaires impliquant un certain profit politique pour le maire.

Conclusion

La mise en évidence de qualités sur des champs d'action possédées par les élus constitue une démarche originale pour caractériser le maire entrepreneur politique. En complément de certains travaux existants [Le Duff R. et Orange G., 1996], cette méthode permet de positionner concrètement l'action des dirigeants politiques municipaux en tenant compte à la fois des dimensions politiques et managériales. II convient néanmoins de souligner les limites de cette étude. Ainsi, la méthode employée ne restitue qu'une photographie à une date donnée de la situation des maires urbains français. Les résultats n'ont donc de valeur qu'à cet instant, même s'il semble que les réponses collectées soient d'actualité pour la durée du mandat des répondants.

Au-delà de ces limites, la typologie des MEP trouve un réel intérêt en matière de conseil managérial auprès des maires, s'inscrivant clairement dans une optique de développement du management public local [Huron D. et Spindler J., 1998]. D'abord, la grille d'analyse de cette typologie peut être affinée et adaptée aux maires et plus généralement aux élus de collectivités locales. En outre, le positionnement du maire sur cette grille peut permettre à ce dernier de se situer non seulement par rapport à sa situation personnelle (court-il des risques?), mais aussi par rapport à ses homologues.

En s'intéressant aux trois dimensions constitutives de la fonction, il apparaît qu'une très forte proportion des élus encourent des risques sur au moins un des champs d'action. C'est sur le champ de l'équilibre politique que les maires 
semblent les plus nombreux en situation de risque (près de sept d'entre eux sur dix). Les conséquences directes peuvent aller jusqu'à la non-réélection du maire sortant. Les risques encourus sur les deux autres axes se traduisent également in fine en risques électoraux dans la mesure où les administrésélecteurs peuvent montrer leur mécontentement lors des votes. Sauf cas particulier et partant du principe que la poursuite du projet ainsi que la pérennité de l'action constituent des objectifs prioritaires pour les maires, leur réélection s'impose alors comme la condition nécessaire mais non suffisante pour atteindre leurs objectifs. 


\section{ANNEXE 1 \\ Construction des cartes de champs d'action des maires}

Afin de déterminer une typologie, il est nécessaire de positionner chacun des maires qui ont répondu en fonction des champs de l'équilibre politique, organisationnel ou en termes de partenariat. Dès lors, l'étude de ces champs d'action du maire se traduit concrètement par la construction de cartes factorielles faisant apparaître deux zones signifiant la possession ou non de la qualité 1 .

\section{La carte du champ de l'équilibre organisationnel}

La construction de cette carte prend en compte six variables : l'introduction d'une modification dans la structure organisationnelle, l'existence d'un projet de commune, l'introduction d'innovation dans l'organisation municipale et la gestion, l'introduction d'innovation dans les relations avec les partenaires, le changement de structure organisationnelle et périodicité de rencontre entre le maire et le secrétaire général.

Les deux axes de cette carte 2 représentent le degré de dynamisme du maire et la nature de ce dynamisme. Les modalités sont regroupées selon deux tendances : un profil de maires caractérisés par une absence de dynamisme, d'innovation, de changement ou de projet et un profil de maire soulignant au contraire un certain dynamisme (agent de déséquilibre). Les observations correspondant à ce profil possèdent donc d'ores et déjà l'une des composantes du maire entrepreneur politique. Notons par ailleurs que ces maires agents de déséquilibre instaurent plutôt un climat de confiance avec leur secrétaire général dans la mesure où ils ne le rencontrent pas souvent et lui apportent ainsi une marque de confiance.

\section{La carte du champ de l'équilibre politique ${ }^{3}$}

Pareillement à la construction précédente, six variables ont été prises en compte pour construire la carte du champ de l'équilibre politique. Les actions des maires s'inscrivant dans le cadre du mandat en cours, les investissements visant des équipements de proximité, ou des priorités des maires axées sur le cadre de vie, la rénovation ou l'emploi, constituent un premier volet d'analyse. Les infrastructures consacrées aux entreprises ou à l'émergence de nouveaux marchés, la communication destinée aux

1 Le positionnement de chacune des modalités sur la carte factorielle, a permis en utilisant une méthode de classification automatique de distinguer deux classes d'observations.

2 représentant $57,6 \%$ de la variance

3 La somme des axes ne représente que $44,5 \%$ de la variance expliquée. Néanmoins, comme le soulignent Jacques Igalens et Patrice Roussel, il est fréquent en Sciences de Gestion de voir le niveau de la contribution factorielle minimale de $50 \%$ ramené à $40 \%$ [1998, p.53]. C'est en ce sens que nous considérons cette dernière comme satisfaisante, d'autant que le troisième axe factoriel ne représente que $12 \%$ de l'explication des variances. 
entreprises et la priorité accordée au développement économique, soulignent un second thème présent sur la carte. L'axe horizontal de cette analyse factorielle décrit l'opposition entre long terme et court terme, alors que c'est l'antagonisme entre activité et passivité qui caractérise l'axe vertical. On peut constater deux tendances.

Une première tendance décrit les maires prenant en compte leur environnement politique. Ce type d'élu privilégiera aussi bien les investissements de proximité que les infrastructures destinées à répondre aux besoins des entreprises et des marchés. Dans ce cadre, l'action prioritaire concernera à la fois le développement économique et le cadre de vie de la population. Leur action est en outre dictée par le besoin de dépasser le mandat en cours. En d'autres termes, le maire ressent le besoin de marquer de son empreinte son passage à la tête de la commune. Le management de l'agenda politique constitue une des dimensions constitutives du concept de maire entrepreneur politique. La seconde tendance met au contraire en évidence un manque de prise en compte de l'environnement politique. L'action du maire est ici encadrée par les contraintes du mandat de six ans et de manière générale il gérera le quotidien sans tenir compte des avis extérieurs. La limitation des investissements et l'absence de priorité affichée complètent en outre la description de cette classe d'observation.

\section{La carte du champ du partenariat}

Pour construire la carte du champ du partenariat des maires ${ }^{1}$, cinq types de variables ont été isolés. La démocratie participative active constitue la première d'entre elles. Elle regroupe l'existence de mairies-annexe, la mise en place de comités de quartiers, de commissions extra-municipales, de comités consultatifs ou la désignation d'élus relais de quartier. La démocratie participative plus ponctuelle, autre variable du champ, comporte l'organisation de réunions publiques et l'utilisation d'enquêtes et de sondages. Ensuite, l'existence d'une structure intercommunale de type intégrée (district, diverses communautés et syndicats d'agglomérations nouvelles) permet également de caractèriser la nature du partenariat. Enfin, la gestion des services par une structure intercommunale ou par délégation de service public est également une variable qui a permis de construire la carte factorielle du champ du partenariat.

Les deux axes de la carte factorielle expliquent d'une part le partenariat que la commune entretient avec les citoyens et les acteurs privés, et d'autre part le partenariat entre communes elles-mêmes. Deux catégories de maires apparaissent. La première proche des modalitès relatives à l'existence d'un partenariat entre le maire, les citoyens, les autres communes et les acteurs privés, décrit un maire naturellement partenaire. La seconde catégorie montre au contraire des élus plutôt réticents à recourir au partenariat.

1 Le pourcentage de variance expliquée est satisfaisant (53\%), et permet d'avancer que cette analyse factorielle est significative. 


\section{REFERENCES BIBLIOGRAPHIQUES}

BECQUART-LECLERCQ Jeanne (1976), Paradoxes du pouvoir local, Paris : Presses de la Fondation Nationale des Sciences Politiques.

BORRAZ Olivier (1994), Le gouvemement des villes: une analyse comparée dans deux villes suisses et deux villes françaises, Thèse de doctorat de Sociologie, IEP Paris.

BOUQUIN Henri (1996), "Pourquoi le contrôle de gestion existe-t-il encore ?", Gestion, vol. 21, $n^{\circ} 3$, septembre, Québec, p. 97-103.

BOUROCHE Jean-Marie et SAPORTA Gilbert (1993), L'analyse des données, Paris : P.U.F.

BUSSON-VILLA Florence (1999), "L'émergence d'une logique évaluative dans la gestion publique : le cas des organisations communales", Finance Contrôle Stratégie, vol. 2, n¹, mars, Paris, p. 5-25.

CHARREAUX Gérard (1997), " L'entreprise publique est-elle nécessairement moins efficace?", Revue Française de Gestion, n¹15, septembre-octobre, Paris, p. 38-56.

CROZIER Michel (1967), La société bloquée, Paris : Le Seuil.

DAHL Robert (1971), Qui gouverne?, Paris: Librairie Armand Colin [traduction française de Dahl Robert (1969), Who governs?, Yale University Press].

DION Stéphane (1986), La politisation des mairies, Paris : Economica.

DURAN Patrice et THOENIG Jean-Claude (1996), "L'Etat et la gestion publique territoriale", Revue Française de Science Politique, vol. 46, $n^{\circ} 4$, août, Paris, p. 580-623.

GIBERT Patrick (1990), "Vers un management des collectivités locales", in Ceram (1990), Le management public, Actes du colloque national des 27 et 28 septembre 1990, Sophia-Antipolis : Association Ceram Management Public, p. 67-76.

GREMION Pierre (1970), "Introduction à une étude du système politicoadministratif local", Sociologie du Travail, $n^{\circ} 1$, p. 51-73.

GUENGANT Alain (1985), "Doit-on gérer une commune comme une entreprise?", Chroniques d'Actualité de la SEDEIS, tome 32, n¹1, novembre, Paris, p. 387-392.

HURON David (1999), Le maire entrepreneur politique; analyse dans les communes de plus de 20000 habitants, Thèse de doctorat de Sciences de Gestion, Université de Nice-Sophia Antipolis.

HURON David et SPINDLER Jacques (1998), Le management public local, Paris : L.G.D.J.

IGALENS Jacques et ROUSSEL Patrice (1998), Méthodes de recherche en gestion des ressources humaines, Paris : Economica.

KLOPFER Michel (1986), "Gérer une commune comme une entreprise ?", Revue Harvard l'Expansion, $n^{\circ}$ 40, Printemps, Paris, p. 93-98.

LE DUFF Robert et ORANGE Gérald (1996), "Essai de typologie du Maireentrepreneur des grandes villes françaises", in Le Duff Robert et Rigal JeanJacques (éd.), Le Maire-Entrepreneur ?, Pau : Presses Universitaires de Pau, p. 36-71

LE DUFF Robert et RIGAL Jean-Jacques (éd.) (1996), Le MaireEntrepreneur?, Pau : Presses Universitaires de Pau.

MARFAING Olivier et SPINDLER Jacques (1998), "Les banques et le risque collectivités locales", in Spindler Jacques (études coordonnées par), Contrôle 
des activités bancaires et risques financiers, Paris : Economica, p. 315335.

MAURY Yann (1997), "Les contradictions du néo-libéralisme gestionnaire: l'exemple du système municipal nîmois (1983-1995)", Politiques et Management Public, vol. 15, $n^{\circ}$ 4, décembre, Paris, p. 145-170.

MOE TERRY M. (1995), "The politics of structural choice : toward a theory of public bureaucracy", in Williamson Oliver E. (éd.), Organization theory. From Chester Barnard to the present and beyond, New-York, Oxford: Oxford University Press, p. 116-153.

ORANGE Gérald (1992), Les stratégies non budgétaires des villes, Caen : Thèse de Doctorat de Sciences de Gestion.

PADIOLEAU Jean-Gustave (1986), L'ordre social, principes d'analyse sociologique, Paris : L'Harmattan.

PADIOLEAU Jean-Gustave (1999), "L'action publique post-moderne: le gouvernement politique des risques", Politiques et Management Public, vol. $17, n^{\circ} 4$, décembre, Paris, p. 85-127.

PAYEN Guillaume (1987), "Les dynamiques de la décision : le plan "câble" et ses entrepreneurs politiques", Politiques et Management Public, vol. 5, n², juin, Paris, p. 65-90.

SALANCIK Gèrald R. and PFEFFER Jeffrey (1977), "Constraints on administrator discretion, the limited influence of mayors on city budgets", Urban Affairs Quaterly, vol. 12, $\mathrm{n}^{\circ}$ 4, June, p. 475-498.

THOENIG Jean-Claude (1975), "La relation entre le centre et la périphérie en France - Une analyse systémique", Bulletin de I'IlAP.

THOENIG Jean-Claude (1995), "De l'incertitude en gestion territoriale", Politiques et Management Public, vol. 13, n³, septembre, p. 1-27.

THOENIG Jean-Claude et BURLEN Katherine (1998), "Les secrétaires généraux des villes", Politiques et Management Public, vol. 16, $n^{\circ} 1$, mars, 141-172.

VERAN Lucien (1991), La prise de décision dans les organisations, Paris : Les Editions d'Organisation.

VIEILLEVILLE Jacques (1994), Les risques de gestion des collectivités territoriales, Paris : Dalloz.

WACHTER Serge (1995), La ville contre l'Etat ?, Montpellier : GIP-Reclus. WACHTER Serge (1998), Economie politique de la ville, les politiques territoriales en question, Paris, L'Harmattan. 